\title{
Protective effect of Curcumin on chronic cerebral ischemia by altering expression of $\alpha$ - synuclein in 2VO model
}

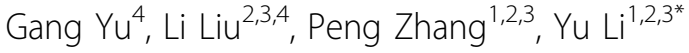 \\ From 2011 International Conference on Molecular Neurodegeneration \\ Shanghai, China. 22-24 September 2011
}

\section{Background}

Previous studies have shown that natural compound Curcumin can improve some biological effects induced by chronic cerebral hypoperfusion and may represent a target for treatment. $\alpha$ - synuclein oligomerization and aggregation are considered to have a role in the pathogenesis of brain ischemia/reperfusion. However, the effects of Curcumin on $\alpha$ - synuclein in chronic cerebral hypoperfusion are poorly understood. This study aims to observe the effect of Curcumin on chronic cerebral ischemia model in rats and investigate change of $\alpha$ synuclein induced by Curcumin.

\section{Methods}

The chronic cerebral ischemia was produced in male Sprague-Dawley rats by permanent occlusion of bilateral common carotid arteries (2VO). Animals were randomly divided into 5 groups: normal control group, shamoperated group, $2 \mathrm{VO}+\mathrm{DMSO}$ group, $2 \mathrm{VO}+\mathrm{Curcumin}$ $100 \mathrm{mg} / \mathrm{kg}$ group, $2 \mathrm{VO}+\mathrm{Curcumin} 50 \mathrm{mg} / \mathrm{kg}$ group. After surgery, all animals were injected intraperitoneally with DMSO solution of Curcumin or a same volume of normal DMSO. Each group was injected once daily for four consecutive weeks. After the completion of the behavioral testing, rats were sacrificed. Hematoxylin-eosin staining and Nissl staining were carried out in section. The expressions of $\alpha$-synuclein protein in hippocampus were detected by immunohistochemistry.

\section{Results}

The chronic cerebral ischemia in rats resulted in a significant pathological change in the hippocampus CA1 area, including: loss of pyramidal cell, shrinkage of nuclei, dark staining of neurons, loss of Nissl body and glial proliferation as compared with sham-operated rats. The administration of different doses of Curcumin attenuated neuronal injury in rats induced by chronic cerebral ischemia along with the concomitant increased the numbers of $\alpha$-synuclein -positive cells.

\section{Conclusion}

Our data demonstrated that the neuroprotective effect of Curcumin involved in increasing the protein levels of $\alpha$-synuclein induced by ischemia.

\section{Acknowledgments}

This study was supported by the National Natural Science Foundation of China (No.30973154), Chongqing Science and Technology Commission Foundation (No.2009BB5270), and Chongqing Municipal Education Commission Foundation (No.KJ090301).

\section{Author details}

${ }^{1}$ Department of Pathology, Chongqing Medical University, China. ${ }^{2}$ Institute of Neuroscience, Chongqing Medical University, China. ${ }^{3}$ Chongqing Key Laboratory of Neurobiology, Chongqing Medical University, China. ${ }^{4}$ Department of Neurology, The First Affiliated Hospital, Chongqing Medical University, Chongqing, 400016, China.

Published: 7 February 2012

\section{doi:10.1186/1750-1326-7-S1-S33}

Cite this article as: Yu et al:: Protective effect of Curcumin on chronic cerebral ischemia by altering expression of $\alpha$ - synuclein in $2 \mathrm{VO}$ model. Molecular Neurodegeneration 2012 7(Suppl 1):S33.

${ }^{1}$ Department of Pathology, Chongqing Medical University, China

Full list of author information is available at the end of the article

(c) 2012 Yu et al; licensee BioMed Central Ltd. This is an Open Access article distributed under the terms of the Creative Commons 